\title{
A CASE OF CATARACTA ELECTRICA, EXAMINED WITH GULLSTRAND'S SLIT-LAMP
}

BY

\author{
Harald G. A. GJessing
}

DRAMMEN, NORWAY

CASES of cataract, resulting from stroke by lightning or hightension electric current, may not be so rare as generally imagined from the few cases mentioned in the medical literature of the world. This is possibly due to the fact that about 90 cited cases have been so minutely examined that nothing new could possibly be brought out. In Gullstrand's slit-lamp, however, we have a new and very efficient means of facilitating ophthalmological diagnosis. According to my knowledge only 3 cases of cataract by lightning have so far been examined in this way, this having been done by Professor Koeppe in Halle, who, with Professor Vogt in Basel, is our greatest authority on slit-lamp microscopical examinations. As my findings, however, in some respects differ from those of Professor Koeppe, the publishing of the account below may have its justification.

On September 5, 1920, a-12-year-old boy, B. L., climbed an iron mast supporting the high-tension wire from Ljabro to Drammen. About $\frac{3}{4}$ metre from the top he was short circuited by the 50,000 volts current and remained suspended on the mast in a state of paralysis. Not until 10 minutes later was he taken down unconscious. In the regular way revival exercises were applied. A little more than an hour later he was brought to Drammen Hospital, where the following state was observed :-

The boy is well built, but pale. Nothing wrong with the lungs. A subsequent examination of the urine showed neither albumen nor sugar.

The entire region from above the left upper eyelid to below the left clavicle is the location of a third degree burn. A temporary examination of the eyes, as also a later and more exact one, during his stay in hospital, showed no intraocular changes.

On the left leg, just on the outside of the knee cap, as also on the outside of the left nates, is seen a $5 \times 5$ centimetre round ulcer with dirty, grey, partly ragged edges, this ulcer going straight down to the bone.

The patient stayed in hospital from November 5, 1920, to February 7, 1921. A number of transplantations were performed ad modum Thiersch on neck, nates and leg. When leaving hospital the wounds were nicely healed. The scars were smooth, 
and only a distortion of the left corner of the mouth marred his looks. The eyes were entirely normal.

During the spring his sight, especially in the left eye, decreased. He came to my office the first time on November 21, 1921. Sight in the left eye was then reduced to finger-counting at 5 metres distance, and in the right eye to $5 / 12$. Glasses did not improve it.

Through further examination of the eyes with Gullstrand's slitlamp and Czapski-Zeiss corneal microscope the following was found in using from 20 to 80 times magnification :-

O.S. With focal illumination the lens gives an even grey reflex. Under the rays of the slit-lamp and from 20 to 80 times magnification the hazy cortex, located immediately below the capsule, is noticed to have an appearance like a loofah sponge. In every direction thicker and thinner asbestos-looking, grey-white, fibretrends are crossing each other. They are of different thickness and follow nowhere the direction of the normal lens-fibres. In the meshes between these fibre-trends are seen, partly, close, fine opacities of a round or polygonal shape; partly, round, vacuolelike empty cells, whose walls are made from close, grey trends. In the central part of the lens surface the opacities are fuller than in the peripheral parts. The deeper layers of the lens cannot be studied in detail owing to the opacities.

O.D. With focal illumination and direct ophthalmoscopy a few peripheral opacities of the type described by Vogt under the name of cataracta coronaria are seen in homatropin-mydriasis, both in the front and the rear cortex. Fundus is seen normal, only obscured. The delineations, however, are a little blurred, due to the lens opacities, as plainly appears under the slit-lamp. Scattered around in the lens capsule itself are a number of round, refractive cysts of different size. It remains to be said that the capsule itself and the lens layer immediately below it is the location of a fine, grey veil, especially so in the central parts of the surface, thus partly blurring the lens shagreen. At 80 times magnification this veil is seen to consist of innumerable fine threads, crossing each other in all directions, and resembling silver filigree. They do not in any place follow the run of the normal lens fibres. In this fine meshwork a few fine, greatly glittering points are located. A thin lens layer below this network was entirely homogeneous.

In the deeper layers of cortex are seen a few corkscrew-like twisted and twined ribbons partly branched and of different thickness. They have different courses and are transparent to such extent that they cannot be seen with the magnifying mirror, but only with the slit-lamp and about 80 times magnification. The most central parts of the lens-corresponding to the centre of Vogt's embryonal nucleus-are apparently quite unaffected. In 
the posterior pole region of the capsule or the cortex immediately below this are a few polygonal, flat or cup-shaped stains.

The tension of the eyes is normal ; $5,5 / 5$. The vision and the mobility are normal.

At a later examination, February 28, 1922, mainly the same observations were made. The sight, however, in the left eye was reduced to finger-counting at a distance of 2 metres, and in the right one to $5 / 15$. The opacities in the right eye were closer together so that the fundus could be seen in reversed picture only with difficulty. While the anterior chambers at the first examination were of a normal depth, they were now shallow, especially in the right eye. Sight was normal. The urine contained neither albumen nor sugar.

The first account of cataracta electrica was published by $\mathrm{Th}$. Saemisch in 1864 in Klinische Monatsblätter f. Augenheilkunde. A one-sided, rapidly increasing, grey cataract developed in the case of an 18-year-old girl 5 months after she had been struck by lightning. Leber could, in 1882, gather 6 cases from the literature of the world, where cataract developed from half a year to one year and a half after each person had been struck by lightning. A number of additional cases were now published, quickly succeeding the above mentioned. It was also shown that a powerful electrical discharge, striking the head, might develop identical results. In Norway a similar case has been published by Dr. Scheen (1906) in "Tidsskrift for den norske Laegeforening," under the heading: "How powerful an electric current can a person stand without being killed?" In this case the person in question had been struck by about 6,000 volts in the head, which became the seat of a severe burn, as big as a hand, and pentrating down to the bone. About 4 months later a right-sided increasing cataract commenced developing, maturing after 2 years. This was examined by Dr. Holth, who points out in his description that the cataract, before maturing, formed an irregular, grey lattice-work of twined and branched ribbons, both in the front and the rear cortex, more, however, in the latter, and never following the normal structure of the lens.

The first observer to deal experimentally with eye injuries caused by electricity, was v. Hess. Experimenting on rabbits and cats, he let their orbital regions be struck by sparks from big Leyden jars. Subsequently a high degree of miosis and iris anaemia was developed; later on, sometimes merely after half an hour to a couple of hours, streak chemosis and opacity of cornealepithelium would develop, and the anterior chamber would be filled with exudation as hyperaemia of iris and ciliary bodies appeared. After 2 to 4 hours he could localize fine, grey opacities in the equatorial regions of the lens. These might 
disappear again or increase, so that the lens during $1 \frac{1}{2}$ to 4 weeks might acquire the characteristics of a cataract. The first microscopical change detected by v. Hess, even as soon as $\frac{1}{4}$ to $\frac{1}{2}$ hour after the eye had been injured, was an extensive dying off of the epithelium on the front capsule, similar to the one appearing after the experimental naphthalene-and massagecataract. A series of minute works has been done by different persons in order to find out how and in what way this change of the epithelium and fibres of the lens occurs after the electrical shock, without, however, it being settled, as yet. That the ciliary body in one way or another has to do with it would seem certain, according to investigations by v. Hess, Knies, Silex, Vossius, Hamburger, and recently Löwenstein.

If, according to this summary, we look at the above account, we immediately notice the peculiar shape of the opacities. It would seem, drawing conclusions from my case, as also from a number of others referred to, that the characteristics of the opacities with cataracta electrica are the confusion of corkscrew-shaped threads and laces, crossing each other in all directions, without following the regular run of the lens fibres. This, which is even seen using ordinary focal light, is beautifully distinct when seen under Gullstrand's slit-lamp. These ribbons may be of very different thickness in more developed cases of cataract. In the first stage-as in the right eye in our case-the threads had a veillike appearance, located immediately below the capsule and partly blurring the lens-shagreen. Also in the deeper layers they formed broader ribbons, extremely twined and interlaced. In the mesh openings between these ribbons and silver glistening threads, fine and very glistening points were located. Vogt holds that these are cholesterin, while Koeppe does not want to express any opinion on this point. I am sorry to state that I have not had an opportunity by chemical analysis after operation to add to the solution of this question.

Further, it is to be seen that with cataracta electrica innumerable refractive arch-shaped cysts in the anterior capsule respectively underlying the capsular epithelium are formed. I have seen a similar picture only with glaucomatous and traumatic cataract. Koeppe states that it is also seen with complicated cataract. These peculiar cysts, giving the surface of the lens the very same appearance as that seen under the rays of the slit-lamp in vesiculosa externa must be supposed to result from dead epithelium, forming a liquid albuminous mass. I refer to the description given by v. Hess about the histological type of lightning cataract in rabbits and cats in Graefe-Saemisch Handbuch $d$. Ges. Ophthalm., third edition, 1911. This histological type, developed experimentally, is shown "in vivo" through the slit-lamp. 
In the cases described by Koeppe, as well as in mine, there was a homogeneous layer below the most superficial capsular and cortical opacities, which would seem to indicate that a lamellar cup-shaped separation between the most superficial cortical layers had taken place. The described opacities in the rear layers must be construed in the same way.

In one point the slit-lamp findings for the right eye differ from those published by Koeppe. In our case a few peripheral opacities resembling bicuspid teeth were seen in the front as well as the rear cortex, subcapsularly located in the place characteristic of Vogt's coronal cataract bordering on the extreme and the middle third of the lens radius. As the coronal cataract of Vogt very seldom is seen before the age of puberty, I have only found typical Vogt's cataract in 9 of 177 examined cases below 15 years, not yet having reached the age of pubertyreferring to my work on this subject, based on about 5,000 examined eyes, Kliniske Linsestudier, published in Norwegian 1920 , and reviewed in this journal by Dr. W. G. Sym, pages 178-181 (1921). Of these 9 cases, only 2 were below 10 years, and $\tau$ from 12 to $13 \frac{1}{2}$ years. The possibility is, therefore, not excluded that an electric shock may cause a coronal cataract, even if no single case may serve as proof. Later, slit-lamp examinations will establish this.

Our present case has been unable to render any further assistance in determining what physical-chemical changes are taking place through the application of electric current. In this case there was a high voltage-about 50,000 volts. In one of Löwenstein's 5 cases it amounted to merely 16,000 volts. According to Komoto and Terrien, a discharge of 550 volts is sufficient to cause cataracta electrica in persons so disposed.

Neither 16,000 nor 50,000 volts can have passed straight through the persons hurt, as, according to Scheen, the current used for electrocution in U.S.A. is only a direct shock of 6,000 volts. The insulating air column separating the wire and the victim is of great importance. It is further reasonable to suppose that a person's individual ability or lack of ability to withstand electrical shocks plays an important part in the different cases. 